\title{
A Semi-Dynamic, Game Based and Interference Aware Channel Assignment for Multi-Radio Multi-Channel Wireless Mesh Networks
}

\author{
Maryam Amiri Nezhad* \\ dept. Computer Architecture \\ Universitat Politecnica de Catalunya \\ Barcelona, Spain \\ Fax:34-93-4017055 \\ E-mail: maryam@ac.upc.edu \\ *Corresponding author
}

\section{Llorenç Cerdà-Alabern}

dept. Computer Architecture

Universitat Politecnica de Catalunya

Barcelona, Spain

Fax:34-93-4017055

E-mail: llorenc@ac.upc.edu

\section{Boris Bellalta}

dept. Information and Communication Technologies

Universitat Pompeu Fabra

Barcelona, Spain,

E-mail:boris.bellalta@upf.edu

\section{Manel Guerrero Zapata}

dept. Computer Architecture

Universitat Politecnica de Catalunya

Barcelona, Spain,

Fax:34-93-4017055

E-mail: guerrero@ac.upc.edu

\begin{abstract}
Channel assignment has been extensively researched for multi-radio wireless mesh networks, but it is still very challenging when it comes to its implementation. In this paper we propose a semi-dynamic and distributed channel assignment mechanism called SICA (Semidynamic Interference aware Channel Assignment) based on game theory formulation. SICA is an interference aware, distributed channel assignment which preserves the network connectivity without relying on a common channel nor central node for coordination between mesh routers. SICA applies a real time learner algorithm which assumes that nodes do not have perfect information about the network topology. To the best of our knowledge this is the first game formulation of channel assignment which takes the co-channel interference into account. We have simulated SICA and compared against other channel assignment mechanisms proposed in the literature. Simulation results show that SICA outperforms other mechanisms.
\end{abstract}

Keywords: Channel Assignment; Multi-Radio; Multi-Channel; Wireless Mesh Network; Game Theory; Real-Time Learning;

Biographical notes: Maryam Amiri Nezhad received her MSc in Computer Engineering from Sharif University of Technology (SUT), in 2006. Currently she is doing her PhD in Computer Architecture at the Technical University of Catalonia (UPC). Her areas of interest are mesh networks and channel assignment protocols.

Llorenç Cerdà-Alabern is associate professor at the Computer Architecture Department, Technical University of Catalonia (UPC). He obtained his Ph.D. in Telecommunication Engineering in 2000 from the UPC. His areas of interest include protocols for wireless ad hoc and mesh networks (MAC and routing layers). He has published papers in international conferences and journals and participated in National and EU funded research projects. 
Boris Bellalta received his MSc degree in Telecommunications from the Universitat Politecnica de Catalunya (UPC) in 2002 and the Ph.D. in Information and Digital Communications from the Universitat Pompeu Fabra (UPF) in 2007, where he combined the Ph.D. studies with a full-time assistant professor position. Since 2007, he is a post-doc researcher and full time lecturer at UPF. His main research interests are in the area of traffic engineering, queuing models and wireless communications.

Manel Guerrero Zapata is an Associate Professor (Profesor Contratado Doctor) in the Computer Architecture Department (DAC) at the Technical University of Catalonia (UPC). His research interests include network security, wireless networks, and routing protocols. He is the author of Secure Ad hoc On-Demand Distance Vector (SAODV) routing protocol and of Simple Ad hoc Key Management (SAKM) scheme. Manel Guerrero received his PhD, M.S. and B.S. in Computer Science from the Technical University of Catalonia (UPC) in 2006, 1999 and 1997 respectively. From 1998 to 2003 he worked at the Nokia Research Center in Helsinki (first as Assistant Research Engineer, then as Research Scientist, and finally as Senior Research Scientist). From 2003 to 2005 he worked as an assistant professor at the Pompeu Fabra University (UPF) in Barcelona.

\section{Introduction}

Wireless Mesh Networks (WMNs) are supposed to be the next Internet back-haul providing network connectivity for end users through multi-hop forwarding. However due to the increasing number of devices sharing the same spectrum brand, interference is one of the important factors that can degrade the performance of the mesh networks (Gupta \& Kumar 2000). Interferences can be grouped in two types: external and internal. The external interference appears when two or more coexisting wireless networks work in the same frequency channel (Fig. 1). Although it can be eliminated by using different non-overlapping channels offered in IEEE 802.11 (IEEE-SA 2007), it requires that the different networks agree on the channel distribution. Additionally, as the number of non-overlapping channels is reduced, the scenario where the external interference can be completely avoided is unrealistic. By internal interference we refer to the time overlapping transmissions by nodes of the same network which can result in collisions or transmissions errors. Both external and internal interferences limit the system performance (Si et al. 2010, Ramachandran et al. 2006).

Multi-radio technologies are well known to offer a significant improvement in capacity through the use of multiple channels offered by IEEE 802.11 standards (Draves et al. 2004). The network capacity can be further enhanced if the network employs an intelligent channel assignment which seeks a proper mapping between the available channels and the radios at every node (Raniwala \& Chiueh 2005, Raniwala et al. 2004, Marina et al. 2010).

Channel assignment (CA), was extensively researched but still challenging since many formulations of the problem turn to be NP-hard (Marina et al. 2010, Ramachandran et al. 2006, Si et al. 2010). Considering the time duration between consecutive runs of channel assignment algorithm, many channel assignment approaches fall under the static category, where mesh nodes tune an antenna to a specific channel permanently (see e.g., (Raniwala et al. 2004, Marina et al. 2010, Avallone \& Akyildiz 2008, Amiri Nezhad \& CerdàAlabern 2010) and references therein). Static approaches provide low complexity and low feedback overheads and can achieve performance improvements through minimizing

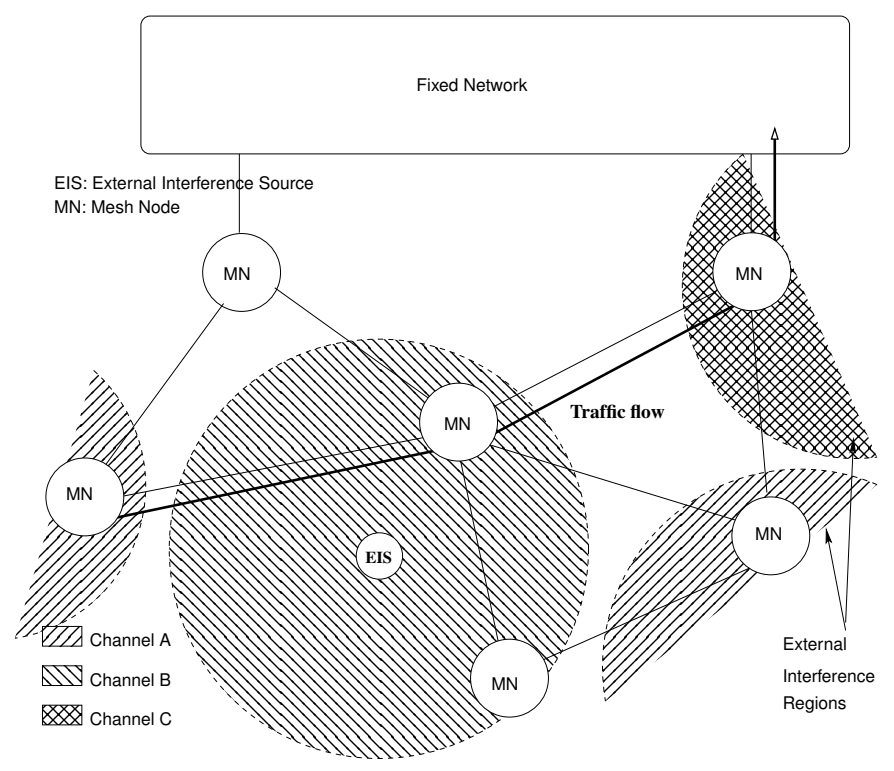

Figure 1: Wireless Mesh Network suffering from interference caused by external sources. If there are only three available channels (A, B and C), to avoid the external interference the mesh network has to be able to find the most suitable channel at each hop.

the internal interference. However due to the variable nature of the wireless medium, the channel assignment mechanism must be flexible enough to adapt to the arbitrary traffic and both external and internal interference patterns (Wu \& Mohapatra 2010).

Dynamic channel assignments (Gong et al. 2009, Bahl \& Chandra 2004), on the other hand, enforce nodes to switch their interface dynamically from one channel to another between successive data transmissions. Therefore they require tight synchronizations among nodes. Dynamic approaches are only applied for single radio nodes working over multiple frequencies, since they can not exploit the advantages of multi-radio networks (Crichigno et al. 2008). 
Static CAs can easily be extended to semi-dynamic by refreshing the channel assignment at regular time intervals in response to changes in traffic pattern or co-channel interference from both external and internal sources (Ramachandran et al. 2006, Wu et al. 2006, Alicherry et al. 2006, Mohsenian-Rad \& Wong 2007, Raniwala \& Chiueh 2005, Kyasanur \& Vaidya 2006, Wooseong Kim \& Gerla 2010, Kim \& Suh 2008, Felegyhazi et al. 2007, Fu \& Agrawal 2008).

In this work we propose SICA, an interference aware channel assignment algorithm for IEEE 802.11 based WMNs. We estimate the amount of interference over channels, induced by any external wireless device, based on IEEE $802.11 \mathrm{k}$ standard (IEEE-SA 2008). We then use game theory to formulate the semi-dynamic channel assignation problem. Unlike previous game formulation in the literature (Felegyhazi et al. 2007, Gao et al. 2008, Chen \& Zhong 2009, Shah et al. 2010, Gao \& Wang 2008) we assume a more realistic scenario by explicitly considering the presence of external interferences from other networks and, we assume that nodes do not have perfect information about others' strategies. Then we apply a real-time learning method to design a distributed algorithm which assigns channels to radios while avoiding the ripple and channel oscillation effects (Si et al. 2010). The nodes continuously refine their decision accounting the changes in the wireless environment. The main contributions that sets our work apart from others are the following ones:

- A novel game theory formulation of the channel assignment problem, considering external and internal interferences.

- A decision making strategy assuming imperfect information at each mesh router that allows a fast network adaption to the changing wireless environment.

- A fully distributed channel assignment algorithm which preserves the network connectivity and supports any routing protocol.

- A new protocol which applies channel load estimation, interface switching, control message exchange and data delivery mechanisms in addition to channel assignment.

We evaluate SICA through simulations using ns-3 (ns3 development team 2011) and compare it with other channel assignment mechanisms that have been proposed in the literature (Wooseong Kim \& Gerla 2010, Ramachandran et al. 2006, Gao et al. 2008). Results demonstrate the effectiveness of SICA in exploiting channel diversity, hence reducing the interference over wireless links and improving the system performance in terms of capacity and supported nodes and networks.

The paper is organized as follows. We first describe the related work in Section 2. Then, in Sections 3-6 we explain SICA architecture, channel assignment algorithm and simulation details. The performance evaluation is done in
Section 7, and Section 8 ends the paper with the concluding remarks.

\section{Related Work}

The channel assignment problem has been studied in deep during last years (Crichigno et al. 2008, Si et al. 2010) and many semi-dynamic solutions have been addressed in previous proposals (Ramachandran et al. 2006, Wu et al. 2006, Alicherry et al. 2006, Mohsenian-Rad \& Wong 2007, Raniwala \& Chiueh 2005, Kyasanur \& Vaidya 2006, Wooseong Kim \& Gerla 2010, Kim \& Suh 2008, Felegyhazi et al. 2007), but few proposals consider the effect of the external interference (Ramachandran et al. 2006, Wooseong Kim \& Gerla 2010).

A simple semi-dynamic approach is proposed in (Kyasanur \& Vaidya 2006) for a mesh network with two radio interfaces per node. Although the authors introduce a new path metric which takes into account the interface switching cost in addition to the expected transmission time (Draves et al. 2004), the proposed mechanism considers only the internal interference.

Breath first search channel assignment (BFSCA) (Ramachandran et al. 2006) is the first interference aware CA mechanism. In BFSCA each node estimates the external interference through monitoring the wireless media and coordinates with a central node through a common channel. The central coordinator then assigns channels to links considering the distance of each link to the coordinator and the quality of the link in terms of transmission delay. The main drawbacks of BFSCA are: it needs tight synchronization between nodes and, the channel assignment algorithm is very slow and time consuming since it does an exhaustive search over all interfering links to find the best channel for each link.

Urban- $\mathrm{X}$ is another adaptive and semi-dynamic channel assignment (Wooseong Kim \& Gerla 2010). Urban-X is proposed for a network where each node must have at least three radios. One radio of all nodes is tuned to a common channel and is used for control traffic. The channel assignment considers the external interference in addition to the number of flows at each node, to make decisions. The best channel is occupied by the receiving radio of a node which has more traffic to send, although it may not receive any traffic.

Channel assignment algorithms using game theory models have been studied recently in some works (Felegyhazi et al. 2007, Gao et al. 2008, Chen \& Zhong 2009, Shah et al. 2010). None of the proposed algorithms considers the effect of co-channel interference. All approaches consider that nodes or players have information about all strategies and payoffs, it means that all nodes make decisions based on a global payoff table. However, in a scenario having external interference, it is difficult to have a perfect knowledge of the channel use, before making decisions.

Felegyhazi et al. (2007), formulate the channel assignment problem as a game where traffic flows compete for shared channels in a conflict situation. Although the algorithm converges to a stable Nash Equilibrium, their work 
is limited to a single hop single collision domain network, where each node participate in only one traffic flow. Further extensions of this work for multi hop networks but limited to one collision domain are presented in (Gao et al. 2008, Chen \& Zhong 2009, Kim et al. 2009), where nodes are limited to communicate with devices in their transmission range. However, although it may be an unrealistic assumption, the authors assume that each node knows about the existence of all other nodes in the network and the channel they use.

Kim et al. (2009) did not put any constraints for the number of radios per node. The proposed game should be played sequentially and channels should be reallocated for any changes in the traffic profile. The approach proposed in (Gao et al. 2008) formulates the channel assignment as a cooperative game where nodes are cooperating with each other to improve the network throughput. The channel reallocation is necessary for any changes in traffic pattern. Shah et al. (2010) formulate the game for multiple collision domains, but they use a static game which is limited to find a Nash Equilibrium for competing flows.

Unlike all previous game models for channel assignment, SICA consider co-channel interference while assuming that nodes have imperfect information and the solution is independent from the traffic profile.

This paper is a revised and expanded version of the workshop paper (Nezhad \& Cerdà-Alabern 2011). A new game theory model that handles better the internal and external interference is presented here. The new game theory model is based on a set of control parameters that improve the system adaptability to the changing environment conditions. Using the new game theory model, SICA achieves a gain equal to $11 \%$ compared with the former protocol (Nezhad $\&$ Cerdà-Alabern 2011). Finally, a new random topology is introduced to evaluate SICA, as well as we extend the protocols to which it is compared (see Section 7.1), showing that SICA outperforms all of them.

\section{SICA Architecture}

We present a multi-radio multi-channel mechanism which mitigates the impact of the interference, and improves the performance of the wireless mesh networks by driving the benefits of non-overlapping channels. The distributed multi-channel architecture considers the channel selection mechanism, describes the switching process of the antennas and controls data buffering and transmitting. Nodes use a distributed algorithm to occupy the best channel based on the information gathered during the channel sensing periods. Channel assignment is viewed as a lower layer mechanism which does not consider the traffic load and, therefore any routing protocol can be applied to the network.

We describe SICA in the specific case where nodes are equipped with two radio interfaces, each one being able to use a set $C$ (with cardinality $|C|>1$ ) of non overlapping channels. However, SICA could be easily extended to a network where nodes are equipped with a number of radios larger than two. The radios will be referred to as the receiving radio and the

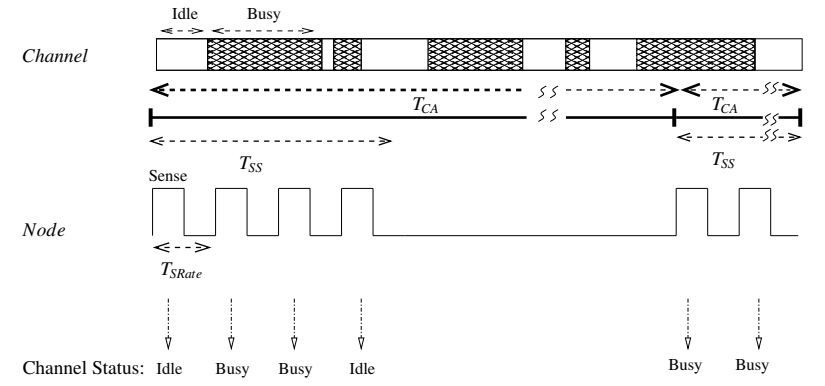

Figure 2: Sensing a channel by gathering samples

transmitting radio, and denoted by $R$ and $T$, respectively (Fu \& Agrawal 2008).

The distributed channel assignment mechanism selects and assigns the best channel to the $R$ radio of each node. Then, nodes switch the $T$ radio accordingly. For example, if a generic node $A$ tunes its $R$ radio over channel $c \in C$, each neighboring node, which aims to send traffic to $A$, will switch its $T$ radio to channel $c$ before start transmission. The $T$ radio remains on channel $c$ until all packets, which are addressed to node $A$, have been sent, or until a maximum period of time, called $T_{\max }$.

In the following sections we explain the details of SICA. We explain the channel sensing mechanism and CA algorithm in sections 3.1, 3.2 and 3.4, respectively. The synchronization and switching of $R$ and $T$ radios are explained in sections 4, 5 and 6.

\subsection{External interference estimation}

To estimate the amount of external interference, mesh nodes use the clear channel assessment (CCA) mechanism for spectrum sensing (Kleinrock \& Tobagi 1975). CCA is based on energy detection during a specific period of time. Since CCA is used to estimate the wireless interference of external devices, to exclude the interference of the nodes from the same network, at a given time all nodes on the same channel stop transmission and start sensing the channel. The required time synchronization for the common sensing period is achieved through sending messages (see Section 4). Since all nodes working on the same channel must remain silent during channel state assessment, a big sensing period will degrade the network throughput. However, a long enough sensing period is necessary to have a precise estimation (Wooseong Kim \& Gerla 2010, IEEE-SA 2008, Nguyen et al. 2011). In SICA, each node senses only one channel at each sensing period (the channel of its receiving radio). Then, at the end of each sensing period, each node exchanges the channel state information with its neighbors.

As shown in Fig 2, during the sensing period $\left(T_{S S}\right)$ every node monitors the channel by taking samples at the sense rate $\left(T_{S R a t e}\right) . T_{C A}$ is the period of time between consecutive runs of channel assignment algorithm.

The channel status would be monitored as either idle or busy. Define $T_{i, b u s y}(c)$ as the time that channel is sensed busy during the sensing period and $T_{i, i d l e}(c)$ as the amount of time that the channel is sensed idle. 
IEEE $802.11 \mathrm{k}$ standard for radio resources measurement (IEEE-SA 2008) proposes a simple formulation to compute the channel load as the percentage of time that the node senses the medium as busy. At the end of the sensing period, node $i$ estimates the normalized bandwidth (or duty cycle) consumed by external networks over a channel $c$, as:

$$
B_{i, n e i g}(c)=\frac{T_{i, \text { busy }}(c)}{T_{i, \text { busy }}(c)+T_{i, \text { idle }}(c)}
$$

The mesh node then uses the channel load to make decision in channel assignment algorithm (see Section 3.2).

\subsection{Game Theory Based Channel Assignment Model}

We use a game theory model for the distributed channel assignment in SICA, which is adaptive to the external interference.

In our model each node is a rational player which tries to occupy the best channel for its $R$ radio. The best channel is a channel which suffers less external interference and it is not shared by many neighboring nodes in the same network. From this point forward, we use the terms node and player interchangeably.

Let $N$ be the number of nodes of the network, and $f_{i, c}$ the number of $R$ radios of player $i$ using channel $c\left(f_{i, c} \in\{0,1\}\right)$. Define the strategy of player $i, s_{i}$, as its channel allocation vector, given by:

$$
s_{i}=\left(f_{i, 1}, f_{i, 2}, \ldots, f_{i,|C|}\right), \quad i=1, \ldots, N
$$

A player strategy describes whether it has a radio over a specific channel or not. Note that the total number of $R$ radios employed by player $i$ is given by

$$
f_{i}=\sum_{k=1}^{|C|} f_{i, k}
$$

In a dual radio network with one $R$ radio for each node, $f_{i}=1$.

We define the strategy matrix (strategy profile), $S$, as the strategy vector of all players at a given time:

$$
S=\left(\begin{array}{c}
s_{1} \\
s_{2} \\
\cdots \\
s_{N}
\end{array}\right)
$$

By $S_{-i}$ we refer to the strategy matrix consisting of all nodes' strategies except player $i$. Note that, node $i$ may not know $S_{-i}$ completely.

We formulate a game theory model where each player $i$ chooses a channel $c$ trying to minimize a loss function. Each mesh router uses two separate costs for selecting a channel. The first cost is according to the channel load estimated in Section 3.1 (Equation (1)). The second cost is according to internal interference induced from neighboring nodes. To estimate the internal interference over a channel, mesh routers compute how congested is the channel in the neighborhood. Let $N_{i}$ be the number of nodes in the interference range of node $i$ (two-hops neighbors based on the interference protocol model presented by Gupta \& Kumar (2000)). We represent by
$R_{i}(c)$ the number of nodes in the set $N_{i}$ that have tuned their $R$ radio to channel $c$ at a given time:

$$
R_{i}(c)=\sum_{k \in N_{i}} f_{k, c}
$$
by

We define the density of interfering nodes over channel $c$

$$
\frac{R_{i}(c)}{N_{i}}
$$

The mesh router then merges the costs by taking the weighted average of the individual cost as a bandwidth loss function:

$$
M_{i, B}\left(c, S_{-i}\right)=\alpha \cdot B_{i, n e i g}(c)+(1-\alpha) \frac{R_{i}(c)}{N_{i}}
$$

where $\alpha \in[0,1]$ is the control parameter.

However, the cost of one node's decision depends not only on the available bandwidth of the selected channel, but also on the switching delay penalty if a node's radio switches frequently (Yang et al. 2011). According to Wooseong Kim \& Gerla (2010), Murray et al. (2007) current 802.11 commodities suffer a considerable switching delay $\left(D_{s}\right)$, that ranges from $80 \mu s$ to $22 \mathrm{~ms}$. We consider the magnitude of the switching delay related to the Hello interval, $T_{H}$ (explained in Section 5).

If the hello interval is large enough the effect of the switching delay is negligible and nodes are allowed to switch frequently to other channels. On the other hand a considerable switching delay should result in a higher channel switching cost, making nodes to switch between channels less frequently.

Let $c_{i}$ the channel being used by node $i$ for the $R$ radio, we assume that a switching delay loss function, for any channel, is given by:

$$
M_{i, D}\left(c, S_{-i}\right)= \begin{cases}\frac{D_{s}}{T_{H}}, & c \neq c_{i} \\ 0, & \text { otherwise }\end{cases}
$$

Finally, we combine bandwidth and switching delay costs in the loss function given by:

$$
M_{i}\left(c, S_{-i}\right)=\gamma M_{i, B}\left(c, S_{-i}\right)+(1-\gamma) M_{i, D}\left(c, S_{-i}\right)
$$

where $\gamma \in[0,1]$ is a tuning parameter. Note that the loss function codomain is $[0,1]$.

It is not feasible nor necessary for a player to compute $M_{i}\left(c, S_{-i}\right)$ for all possible values of $S_{-i}$. Each player computes the loss value for one strategy profile at a time. In Section 3.3 we explain how this method solves the game effectively.

To sum up, we have defined a game with the following properties:

- Nodes are rational players and try to occupy the most vacant frequency channels.

- Nodes do not have knowledge about their neighbors criteria of making decision, beforehand. 
- Each channel decision imposes a cost (in the range of 0 to 1$)$ to a node, as a function of switching delay and available bandwidth on the selected channel.

- The game is played in several rounds, as the external parameters introduced by the environment may differ in each round, the environment is unpredictable and can remain permanently in the transient state.

\subsection{Solving the channel assignment game}

Due to the changes in the co-channel interference, the game outlined in the previous section has no deterministic loss matrix, therefore using common approaches to solve the game is impossible. Our solution is based on real-time learning approach proposed by (Freund \& Schapire 1996, 1999).

As define before, $M_{i}\left(c, S_{-i}\right)$ is the loss matrix of node $i$, i.e. the rows of $M_{i}\left(c, S_{-i}\right)$ are the strategies of node $i$ (the channels, $c \in C$, it can choose), and the columns are all possible strategies of the other players, $S_{-i}$.

Each node assigns non-negative weights $\left(w_{i}(c)\right)$ to the rows of $M_{i}\left(c, S_{-i}\right)$. We assume that, the number of rows in $M_{i}\left(c, S_{-i}\right)$ is the same for all nodes and equal to the number of orthogonal channels $(|C|)$.

Initially $M_{i}\left(c, S_{-i}\right)$ is unknown to player $i$, but this game can be played repeatedly in a sequence of game rounds $(1, \ldots, T)$. To avoid the channel oscillation in each round $t$ $(t \in 1, \ldots, T)$, the player plays a mixed strategy based on the weights $\left(w_{i, t}(c)\right)$ assigned to the rows of $M_{i}\left(c, S_{-i}\right)$. The probability of selecting the channel $c$, is calculated as:

$$
P_{i, t}(c)=\frac{w_{i, t}(c)}{\sum_{c \in C} w_{i, t}(c)}
$$

Initially, all weights are set to 1 , which means that the probability of selecting any channel is identical. After selecting a channel, the node gathers information from its neighbors and updates the loss that is suffered (equation (9)). Then, the weights are updated as:

$$
w_{i, t}(c)=w_{i, t-1}(c) \beta^{M_{i}\left(c, S_{-i}\right)}
$$

where $\beta \in[0,1]$ is the game parameter (Freund \& Schapire 1999). The main theorem (Freund \& Schapire 1996, 1999) concerning this algorithm is:

Theorem 1: For any matrix $M$ with $n$ rows and entries in $[0,1]$, and for any sequence of mixed strategies $Q_{1}, \ldots, Q_{T}$ played by the environment, the sequence of mixed strategies $P_{1}, \ldots, P_{T}$ produced by the algorithm satisfies

$$
\sum_{t=1}^{T} M\left(P_{t}, Q_{t}\right) \leq \frac{\ln \left(\frac{1}{\beta}\right)}{1-\beta} \min _{P} \sum_{t=1}^{T} M\left(P, Q_{t}\right)+\frac{1}{1-\beta} \ln n
$$

The proof can be found in (Freund \& Schapire 1996, 1999). The theorem simply implies that, the excellency of the decisions made by the learner using the multiplicative weights scheme, depends on the value of $\beta$. A high $\beta$ value introduces minor changes to the weights, and the learner follows the environment more accurately but slowly.
Therefore it is applicable to a scenario where the environment changes less frequently. On the contrary, a low $\beta$ value imposes big changes in the weights, which introduces a higher error to the decision but, it is adequate to a scenario with frequent changes. In our simulations we found that $\beta=$ 0.2 leads to better results (see Section 7). We use the same $\beta$ for all players. Note that the best solution reached by the learner is not necessarily the Nash Equilibrium. Since it has been shown that multiplicative weights update learning algorithm cannot work for Nash Equilibrium in general bimatrix games (Daskalakis et al. 2010).

\subsection{Channel Assignment Mechanism}

Alg. 1 summarizes the channel assignment mechanism previously described. Recall that the main idea of SICA is to use all the available information at each node, which is gathered from its neighbors and sensing the channels, and selects the best channel by playing a game with mixed strategies. As explained in Section 3.3, the game is played in rounds that we refer to as channel assignment periods, and represent its duration by $T_{C A}$. Each node $i$ runs Alg. 1 at every $T_{C A}$.

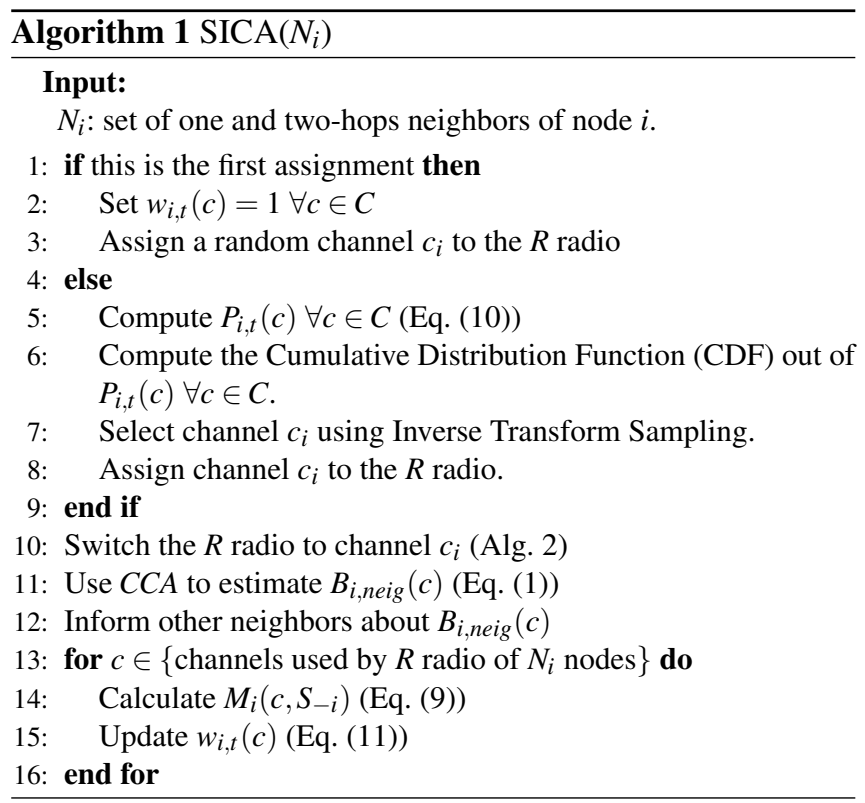

Four main reasons make SICA to be an efficient channel assignment algorithm:

- Nodes are not required to have the perfect information about other players' strategies and loss functions.

- Nodes are supposed to be selfish players trying to occupy the best channels.

- It is not necessary for a node to estimate the external interference over all channels. Each node only estimates the interference over the channel of its $R$ radio.

- The proposed channel assignment eliminates the Channel Oscillation problem. This problem happens 
when some nodes find a channel empty and try to occupy it simultaneously. In such situation, the nodes will switch back as they will find it busy by the others that have switched to that channel too. Playing a mixed strategy, as previously described, avoids channel oscillation since each node selects the destination channel randomly with a predefined probability.

Table 1 summarizes the parameters of the channel assignment mechanism and the default value for each one of them.

\section{Mesh nodes synchronization mechanism}

Unlike most CAs proposed in the literature (see Section 2), in SICA there is not a common control channel shared by all nodes. In SICA, the synchronization is achieved through exchanging messages over the data channels. Since each node can assign a different channel to its receiving $(R)$ radio, nodes must be aware of the channels used by their neighbors' $R$ radios. In SICA, a node broadcasts Hello messages to report the channel of its $R$ radio to its neighbors.

It is not necessary to send Hello messages over all available channels, except when a new node joins the network or when a node stops receiving Hello messages from any neighbor. Once a node knows the channels used by the $R$ radio of its one-hop neighbors, the node switches the $T$ radio to those channels and sends Hello messages every $T_{H}$ seconds.

We refer to the period between Hello messages as $T_{H}$. This period must be long enough to minimize the overhead caused by the switching time of the $T$ radio, and small enough to keep the information updated for all nodes.

A Hello packet contains the following information, besides a sequence number:

1. Channel and MAC address of the $R$ radio,

2. Channels of the $R$ radios of the node's one-hop neighbors,

3. Spectrum sensing information,

4. Channel switching attempt information.

Additionally, each node has to broadcast the the channel used by the $R$ radios of its one-hop neighbors in the Hello messages. Therefore the neighbors are able to keep the information of two-hops nodes.

Spectrum sensing information contains: $i$ ) the estimated consumed bandwidth by external interferences over the receiving channel; and $i i$ ) the time units remaining before the start of the next sensing period. Neighboring nodes need information about the upcoming sensing period to avoid initiating any transmission over the channel that is going to be sensed. Nodes broadcast the remaining time units before their next sensing event periodically, therefore neighboring nodes are informed even though some of the messages get lost. We assume that re-sending the sensing information, which is done frequently via hello messages, reduces the error of miscalculating the sensing time for neighbors.
Channel switching attempt information is the expected time units before the moment that a node will switch its $R$ radio to a new channel (See Section 6). Note that there is no need to have a tight synchronizations between nodes since nodes are aware of changes through Hello messages.

\section{Data delivery mechanism}

After gathering information from the neighbors, a node may start transmitting data. One node may have packets to deliver to different neighbors which have their $R$ radio on different channels. In our model each channel is associated with a sequential first-in first-out (FIFO) queue. Packets are added to the corresponding queue according to the receiving channel of the neighbors. When the $T$ radio switches to each channel, it sends all or some of the packets in the associated queue. We use a different queue for Hello messages, which has higher priority than data packets' queue.

A mesh node uses a Round Robin approach to visit all the channels for which it has data to sent, defined as the subset $C_{i} \subset C$. To avoid starvation, after switching, the $T$ radio will stay in one channel for at most a specific period of time $\left(T_{\max }\right)$. We assume that the switching delay of the $T$ radio is constant and equal to $D_{s}$. Therefore if a node has to send data over $C_{i} \subset C$ channels, it will take $\left|C_{i}\right| T_{\max }+\left(\left|C_{i}\right|-1\right) D_{s}$ to visit them. In order to have the same opportunities to transmit over $C_{i}$ channels, the node computes $T_{\max }$ as:

$$
T_{\max }=\frac{T_{H}-\left(\left|C_{i}\right|-1\right) D_{s}}{\left|C_{i}\right|}-D_{t}
$$

where $T_{H}$ is the period between Hello messages and $D_{t}$ is the delay before the $T$ radio starts the transmission. Note that $C_{i}$ is given by the number of different channels used by node's neighbors.

Fig. 3 shows an example describing the whole process. In the example the node has $\left|C_{i}\right|=2$ channels over which it has data to send. Every $T_{H}$ a Hello packet is pushed at the front of the $C_{i}$ queues. We show Hello and Data packets with black and shaded boxes, respectively. $T_{\max }$ shows the maximum duration of time that a node may remain in each channel (see Equation (13)). In detail, Fig. 3-a shows the node status before $T_{H}$ starts. The mechanism starts from channel one (Fig. 3-b) and, after $T_{\max }$ it switches to channel two, where it is able to transmit only three packets before to switch again to channel one.

After switching the $T$ radio to a channel, to avoid collision with any ongoing transmission on the channel, a node must wait at least for $D_{t}$ units of time before it starts transmitting, (see Section 6.2). After $D_{t}$, the node sends packets during at most $T_{\text {max }}$, then it switches the $T$ radio to channel two and the transmission process is repeated for this channel (Fig. 3-b). While there are packets in the queues, the node will round robin among them until the end of $T_{H}$ (Fig. 3-c).

Note that the $T$ radio can not switch to a channel and initiate any transmission when the channel is being sensed by any of the neighboring nodes. Moreover nodes must consider the switching attempt of the $R$ radio which is announced to the neighbors before it starts. The switching mechanisms of $T$ 


\begin{tabular}{llll}
\hline Parameter Name & Description & Possible Value & Default \\
\hline \hline$T_{H}$ & Hello interval & $10 \mathrm{~ms}-100 \mathrm{~ms}$ & $20 \mathrm{~ms}$ \\
$T_{C A}$ & Channel assignment interval & $T_{H} \ll T_{C A}$ & $10 \mathrm{~s}$ \\
$T_{S S}$ & Channel sense interval & $T_{H}<T_{S S}<T_{C A}$ & $5 \mathrm{~s}$ \\
$T_{S R a t e}$ & Channel sense rate & $T_{S R a t e} \ll T_{S S}$ & $1 \mathrm{~ms}$ \\
$\alpha$ & Bandwidth loss function tuning parameter & $0 \leq \alpha \leq 1$ & 1 \\
$\beta$ & Channel weight parameter & $0<\beta<1$ & 0.2 \\
$\gamma$ & Loss function tuning parameter & $0 \leq \gamma \leq 1$ & 0.8 \\
$|C|$ & Number of available orthogonal channels & $1<|C|$ & 8 \\
$D_{S}$ & Switching delay of the radio & $80 \mu \mathrm{s}-20 \mathrm{~ms}$ & $300 \mu \mathrm{s}$ \\
\hline
\end{tabular}

Table 1 Channel Assignment Parameters

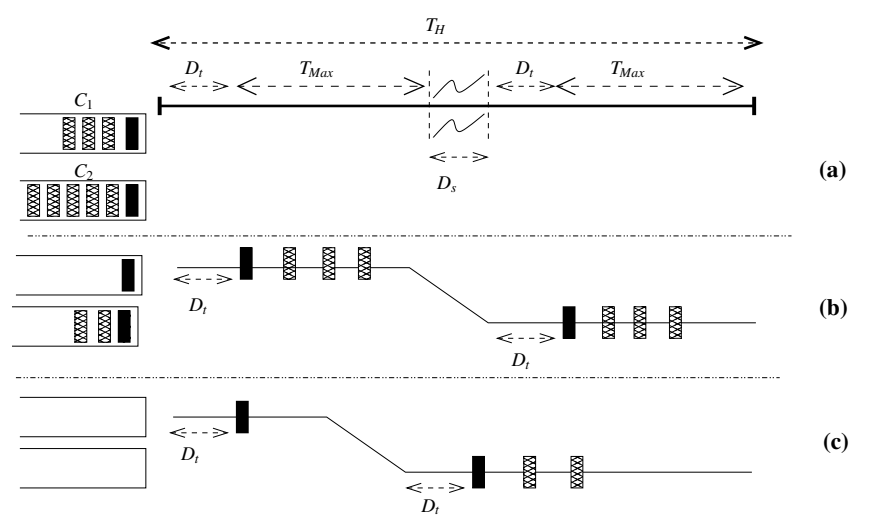

Figure 3: Channel queues and data delivery mechanism

and $R$ radios are explained with more details in the following section.

\section{Channel Switching Mechanism}

The switching mechanism consists of two different protocols for switching $T$ and $R$ radios, respectively.

\subsection{Switching the $R$ radio}

When a node decides to switch the $R$ radio to any channel, it must announce the switching attempt through Hello messages. The switching attempt information in a Hello message, consists the following fields:

1. The destination channel to which the $R$ radio will switch,

2. The time to switch $\left(T_{S}\right)$.

The switching time $\left(T_{S}\right)$ contains the remaining time in the current channel until the $R$ radio switches to the new channel. This time must be longer than the Hello interval to make sure that all neighbors are informed. Therefore the neighbors will consider the new channel for upcoming transmissions. A node follows the steps in Alg. 2 to tune the $R$ radio to the defined channel.

If a node misses any information about a switching attempt of a neighbor, the node would fail to send packets to it. The algorithm tries to prevent this by selecting a

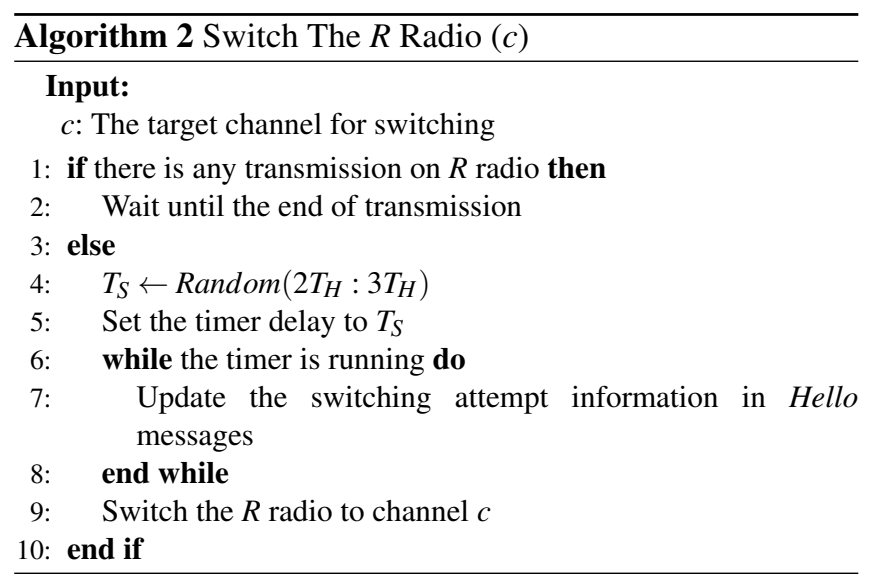

sufficiently large $T_{S}$ (see Table1). Moreover the node always gets information about a lost neighbor from other common neighbors, thus, updating its information.

\subsection{Switching The T Radio}

The $T$ radio switches channels more often than $R$. Alg. 3 describes the switching mechanism for the $T$ radio. Here each node checks all queues sequentially (Round Robin) and if there is any data waiting for transmission, it switches the $T$ radio to the corresponding channel and starts sending data after $D_{t}$ units of time.

When a node switches to a new channel it may fail to hear an on-going transmission between any other nodes on the same channel, so it could be a hidden terminal for the ongoing transmission and must avoid transmitting immediately to prevent the collision. Consequently after switching, a node may wait for $D_{t}$ before starting any transmission.

The node remains on the target channel until the end of the transmission or at most for $T_{\max }$. Then it proceeds to check the other queues.

\section{Performance Evaluation}

In this section, we study the performance of the proposed channel assignment algorithm using ns-3 simulator (ns3 development team 2011) for 802.11-based multi-radio mesh networks. We use a network where the mesh routers initialize their routing tables using Shortest Path First (SPF), 


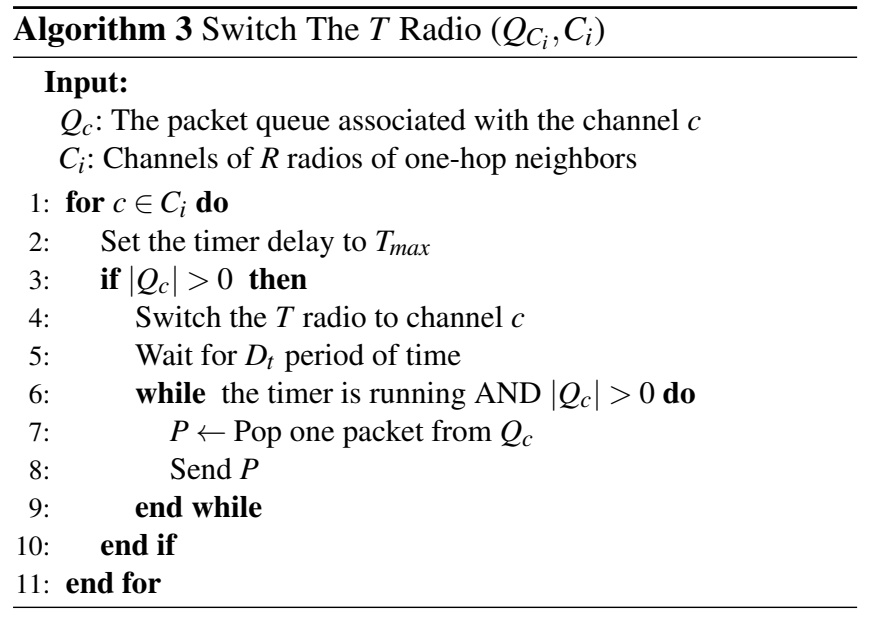

minimizing the number of hops. We assume a two ray ground propagation model with a radio range of $250 \mathrm{~m}$. Wireless nodes can tune their radio to any channel among 8 nonoverlapping channels (according to IEEE 802.11a standard). RTS/CTS mechanism is disabled.

We compare SICA with three relevant channel assignment mechanisms proposed in the literature: the Centralized Breath First Channel Assignment (BFSCA) (Ramachandran et al. 2006); the semi-dynamic interference aware channel assignment (Urban-X) (Wooseong Kim \& Gerla 2010); and the Nash Equilibrium Channel Assignment (NEMCA) (Kim et al. 2009). A baseline single radio network working over one channel is also introduced for completeness.

\subsection{Description of BFSCA, Urban-X and NEMCA}

Breath First Search Channel Assignment (BFSCA) (Ramachandran et al. 2006) is a centralized mechanism that considers one node in the WMN as a coordinator, which is responsible for assigning channels to all nodes' radios in the network. It also considers that one radio at each node is tuned to a common channel for control messages. Each node estimates the external interference through monitoring the wireless media, sending the results of such action to the central node through the common channel. The coordinator then assigns channels to links and informs nodes. Nodes redirect the traffic to the common channel before switching to a new channel and, therefore, they need to be tightly synchronized as, otherwise the channel switching mechanism would interrupt the traffic transmission. Channel assignment in BFSCA uses a graph theory based interference model to find the interfering links (Gupta \& Kumar 2000). It sorts all links based on their distance to the central node and then tries to assign different channels to the interfering links but if such a channel is not found it assigns a random channel to the link.

Urban-X (Wooseong Kim \& Gerla 2010) uses three radios for each node: one $R$ and one $T$ radio, as in SICA, and a third radio which is tuned to a common channel for all nodes. The common channel stays unchanged through the life time of the network. Channel assignment in Urban-X is distributed and takes into account the amount of flows a node has to send, and the estimated external interference over the channels.
Nodes need to have information about the number of flows their neighbors have. Then Urban-X assigns a priority to each node based on the number of active flows it has. Nodes having higher priority have more chances to occupy the best channels. Nodes broadcast control messages over the common channel up to two-hops neighbors. After switching to a channel, the $T$ radio remains there for a predefined period of time (40 ms).

Nash Equilibrium Channel Assignment (NEMCA) (Kim et al. 2009) is a game theory based channel assignment which only considers the internal interference. NEMCA is distributed and played by rational nodes. Nodes consider the internal interference over channels without keeping any memory. Each node selects a list of channels which are better than the current channel it has, from where it selects the next channel to use using a uniform random probability.

\subsection{Simulation Parameters and Performance Metrics}

We have evaluated the performance of the protocols for different number of nodes for two different node placement topologies: grid and random, and with different number of traffic flows. The traffic is generated by $100 \mathrm{kbps}$ CBR flows with packet size of $L=8000$ bits sent over vertical and horizontal directions in the grid topology and between random nodes in the random topology. Data queues are simulated in a way that they drop the old packets automatically to avoid saturation. We assume that the maximum time duration that a packet can remain in a queue is $1 \mathrm{~s}$ for data and $20 \mathrm{~ms}\left(T_{H}\right)$ for Hello messages.

The channels receiving interference from external networks, are chosen randomly. In the simulations, we consider that, at each time $50 \%$ of channels are busy due to the external interference. A channel with external interference is modeled as an on-off process, such that the channel is sensed busy and idle during the on and off states, respectively. Note that as the channel is detected busy due the external interference, nodes are not allowed to transmit during that state. The duration of the busy state has been fixed to a constant value, while the duration of the idle state is chosen exponentially distributed. The duration of the busy and idle periods have been varied to produce different interference loads.

The SICA parameters have been set using the values of Table 1. The specific parameters of other protocols are set according to the values given in (Ramachandran et al. 2006, Wooseong Kim \& Gerla 2010, Kim et al. 2009).

We consider three network performance measures:

- Data delivery ratio: ratio of the total amount of data which is correctly received by the destinations, to the total amount of data packets transmitted by the sources.

- Average end to end delay: mean delay of the packets to reach the destination.

- Control overhead: ratio of the total number of control messages sent between nodes, to the total number of correctly received packets. 


\subsection{Results}

\subsubsection{Grid Topology}

Figures 4-6 show the network performance for different number of nodes and two CBR traffic flows of $100 \mathrm{kbps}$. Every $50 \mathrm{~s}$, external interference is introduced over 4 channels chosen randomly.

In these simulations the duration of the busy state of the external interference is fixed to $10 \mathrm{~ms}$, while the mean duration of the idle state is $8 \mathrm{~ms}$. The results have been obtained averaging over 10 runs of $1000 \mathrm{~s}$ simulation time with different seeds. The error bars in the figures show $95 \%$ confidence intervals.

Fig. 4 shows that the delivery ratio is $20 \%$ higher in SICA and BFSCA than in Urban-X or NEMCA. This is a significant improvement, since Urban-X and BFSCA use 3 radios and SICA uses only 2. This result shows that the game theory approach used in the channel assignment of SICA outperforms the optimized centralized algorithm used in BFSCA for large networks. The bigger error bars in NEMCA and the single-channel network shows that they suffer more unexpected external interference which is avoided by adaptive schemes. Moreover, NEMCA, as a non adaptive channel assignment, performs worse than a single channel network because of the radio switching penalty and channel assignment overheads for making inefficient decisions without considering the external interference.

In Fig. 5 we can see that the average end to end delay is lower in SICA and BFSCA than the other protocols. SICA leads to a lower delay thanks to the fast switching mechanism of the $T$ radio over all channels (see Section 5) and because it avoids the channels which suffer external interference. The average delay in Urban- $\mathrm{X}$ is much higher than others because it keeps the $T$ radio in each channel for a predefined period of time, regardless of having data to send, therefore forcing a considerable delay for data waiting in the queues of the other channels.

Fig. 6 shows that SICA and BFSCA have similar control overhead. BFSCA uses a common channel for control packets which should be forwarded until the coordinator node, in SICA on the other hand a node broadcasts control messages only over all channels which are used by its neighbors. The control overhead in NEMCA is much higher than others since it broadcasts many control messages for channel assignment but has a low data delivery ratio. The control messages of the single channel network consist of Hello messages that nodes send every $T_{H}$ seconds to their neighbors to announce their presence.

\subsubsection{Random Topology}

Fig. 7-9, show the packet delivery ratio, average end to end delay and control overhead for different number of nodes which are randomly distributed over a $1000 \times 1000 \mathrm{~m}^{2}$ area. We check the network topology to be connected using $\mathrm{R}$ numerical tool ( $\mathrm{r}$ development core team 2008). Each point in the figures shows the average over 50 runs. The error bars in the figures show $95 \%$ confidence intervals. Fig. 7 shows

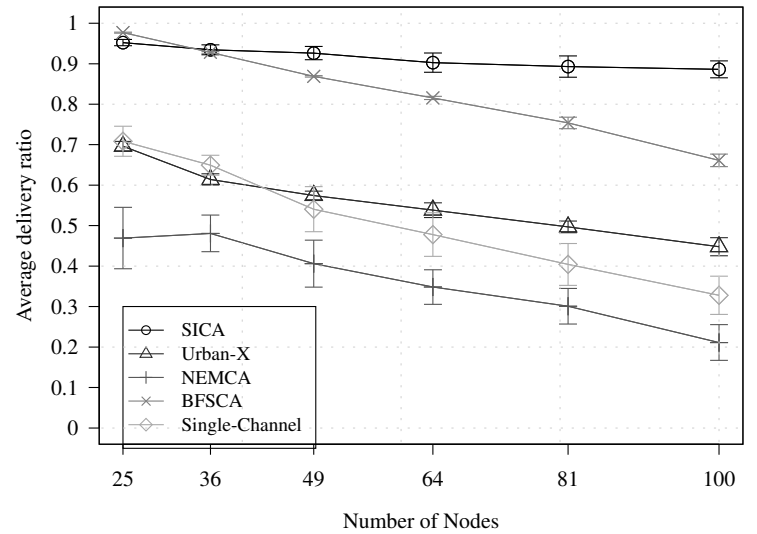

Figure 4: Data delivery ratio vs. number of nodes (Grid topology)

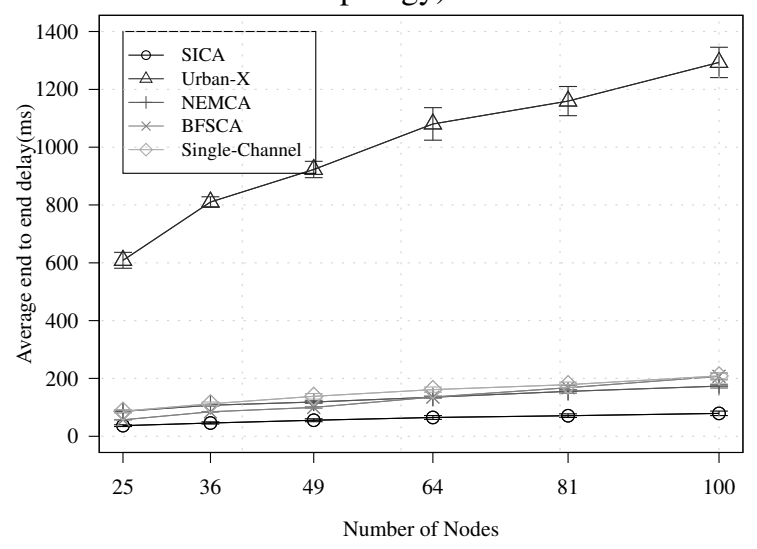

Figure 5: Average end to end delay vs. number of nodes (Grid topology)

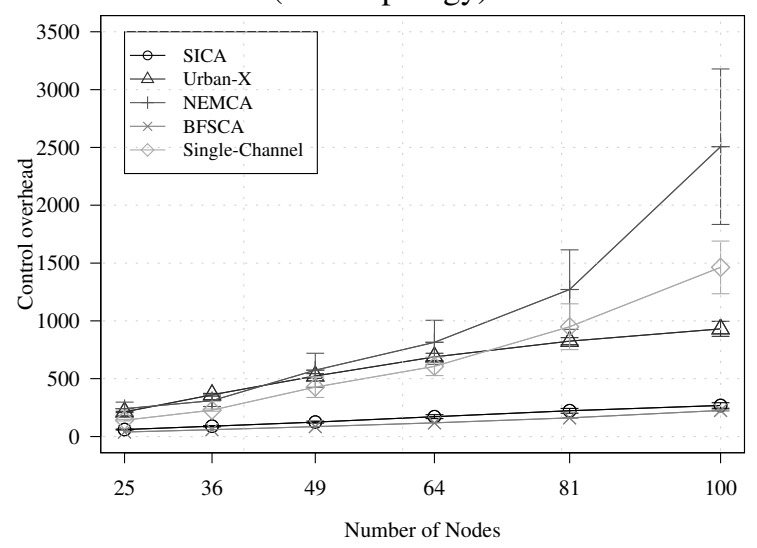

Figure 6: Control overhead vs. number of nodes (Grid topology)

that the delivery ratio in SICA is higher than other protocols and it does not drop a lot as the number of nodes increases. For BFSCA and Urban-x on the other hand, the delivery ratio drops fast as the number of nodes increases. NEMCA performs the same as a single channel network following the reasons explained for Fig. 4.

Fig. 8 shows that the average end to end delay of all protocols is much lower compared to Urban-X due to the same reason explained for Fig. 5.

Fig. 9 confirms the reason explained for Fig. 6, showing that control overhead for NEMCA is higher than other 


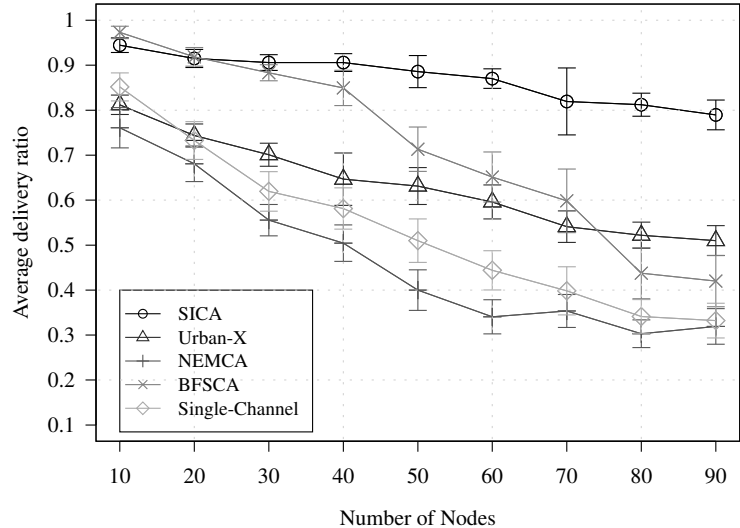

Figure 7: Data delivery ratio vs. number of nodes (Random

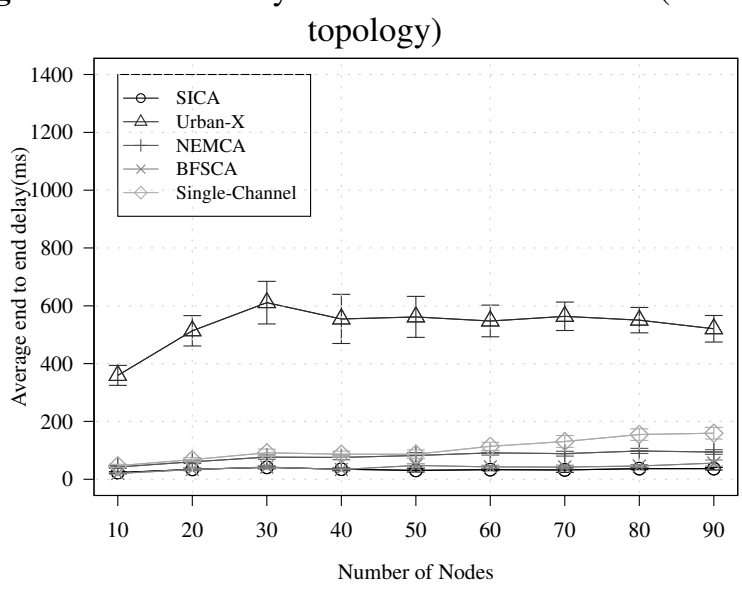

Figure 8: Average end to end delay vs. number of nodes

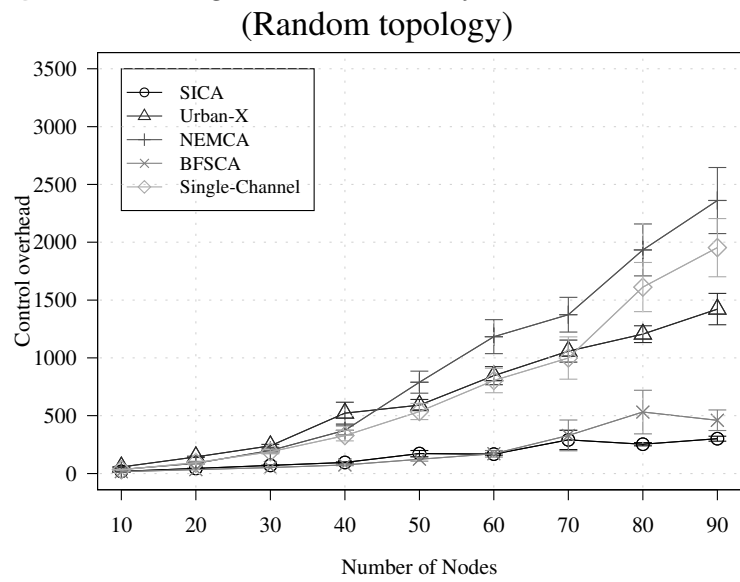

Figure 9: Control overhead vs. number of nodes (Random topology)

protocols, while SICA and BFSCA lead to lower control overhead.

\subsubsection{Increasing the number of traffic sources}

Fig. 10-12 are obtained using a $8 \times 8$ grid network while other parameters are the same as the parameters considered for the grid scenario.

Fig. 10 shows that the delivery ratio of SICA is higher than others in presence of high traffic load. The delivery ratio of BFSCA drops fast increasing the number of traffic flows, since any channel switching interrupts the data transmission and nodes are forced to deliver data packets through common channel which offers a high load over the common channel (Section 7.1).

Urban-X performs better in presence of high load traffic compared to BFSCA since it considers the traffic load for making decision, but it results in a considerable high end to end delay (Fig. 11).

Fig. 12 shows that the control overhead of SICA and BFSCA is almost better than others.

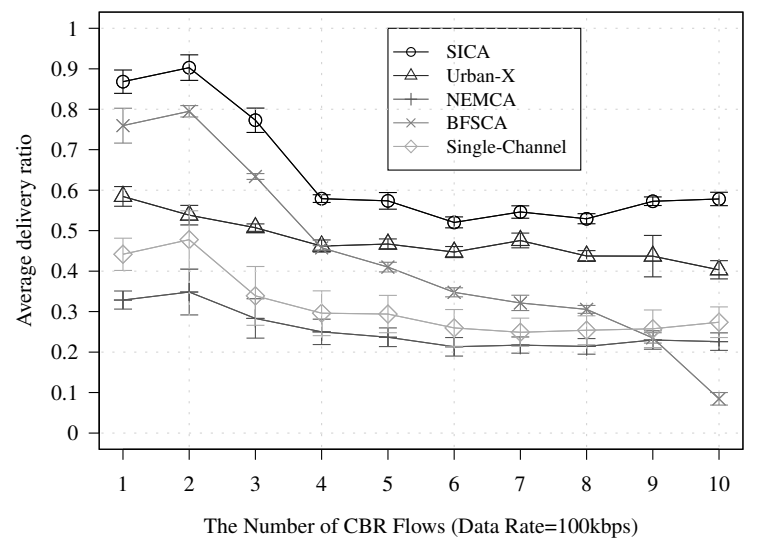

Figure 10: Data delivery ratio vs. number of CBR traffics

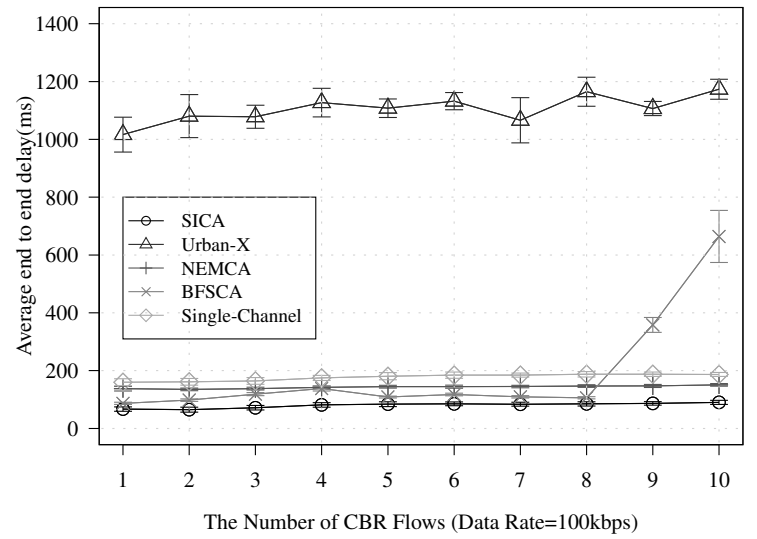

Figure 11: Average end to end delay vs. number of CBR traffics

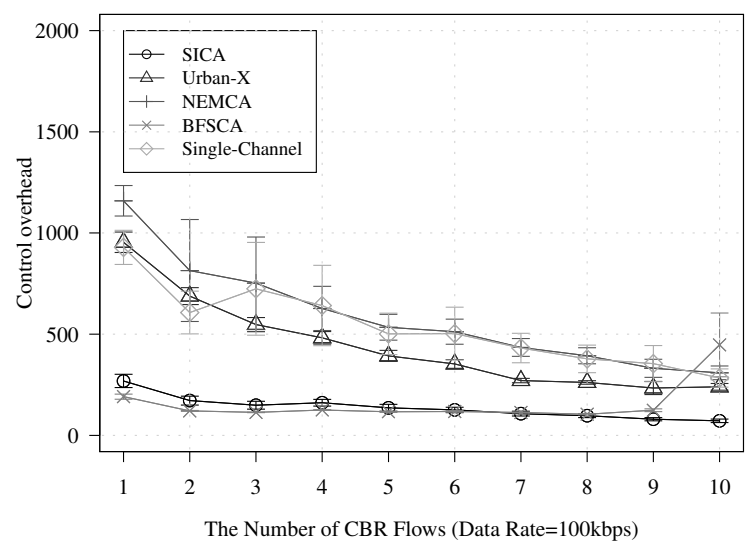

Figure 12: Control overhead vs. number of CBR traffics 


\subsubsection{Changing the interference load}

Fig. 13 is obtained using a $8 \times 8$ grid network while other parameters are the same as the parameters considered for the grid scenario.

Fig. 13 compares the delivery probability obtained with all protocols, varying the load of the interference. The $\mathrm{x}$-axis of these figures shows the amount of the external interference, which is varied by changing the duration of the busy state of the interference process between $5 \mathrm{~ms}$ and $20 \mathrm{~ms}$, and maintaining the mean duration of the idle state equal to $8 \mathrm{~ms}$. We introduced interference over 4 channels. Fig. 13 shows that, even with a high interference load, the delivery ratio in SICA and BFSCA does not change, when the interference is increased. On the other hand, delivery ratio in Urban$\mathrm{X}$ and NEMCA drops fast. The result confirms that, SICA and BFSCA are much more robust and less sensitive to the external interference than Urban-X.

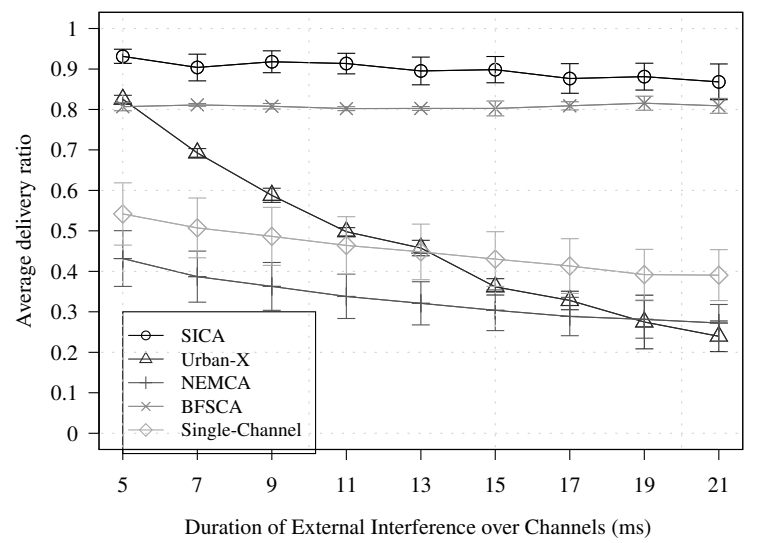

Figure 13: Data delivery ratio vs. Busy duration of external interference

\subsubsection{Data delivery ratio: temporal evolution}

In order to have a more detailed view of the protocol's behavior, Fig. 14 shows the time evolution of the delivery ratio obtained with different channel assignment protocols using a $8 \times 8$ grid network. Other parameters are the same as the parameters considered for the grid scenario. The values shown in the figure have been obtained repeating the simulation for 20 different random seeds and averaging the delivery ratio over $5 \mathrm{~s}$ periods.

Figure shows that in SICA the delivery ratio is kept more stable than in others. BFSCA is also able to offer a high packet delivery ratio with a few variations. Urban-x shows high variations in delivery probability and NEMCA is incapable to avoid the interference over channels.

\subsubsection{SICA performance as function of $\alpha, \gamma$ and $\beta$}

Finally, we investigate the sensitivity of SICA to the $\alpha, \gamma$ and $\beta$ tuning parameters that characterize the response of the game theory model (see Section 3.2). Fig. 15-17 are obtained using a $8 \times 8$ grid network while other parameters are the same as the parameters considered for the grid scenario.
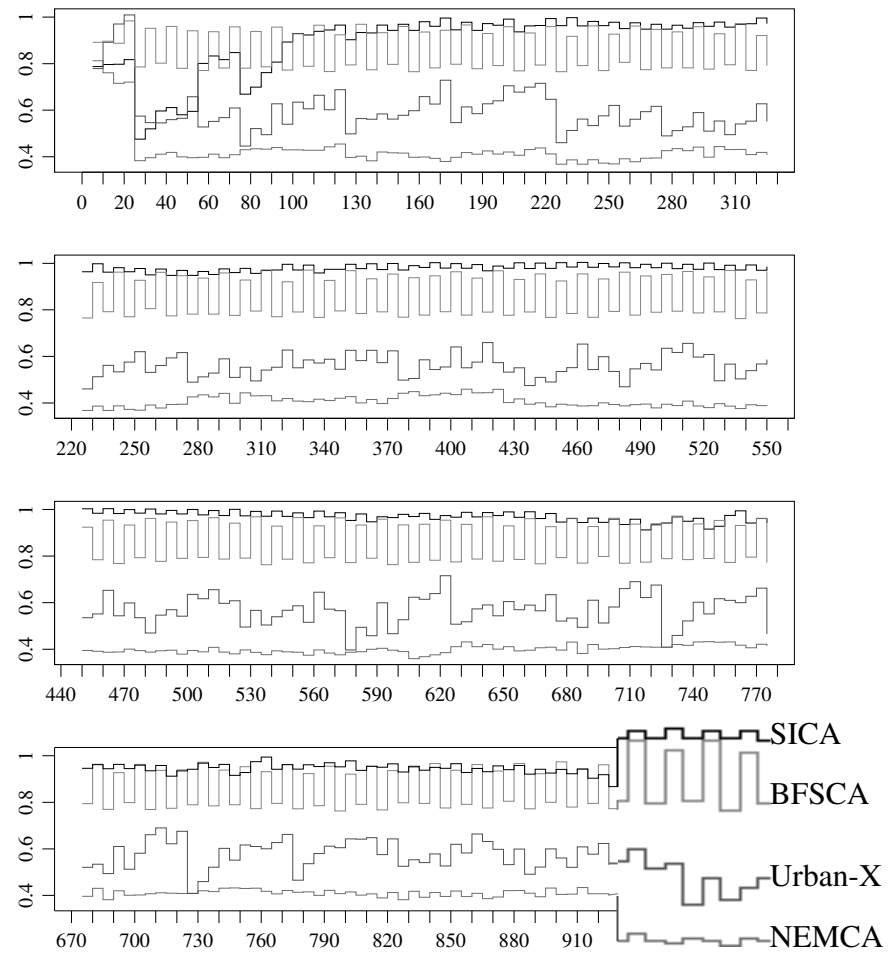

Figure 14: Data delivery ratio over time (zoomed in at the end of the graph in order to make it easier to see which line corresponds to each protocol)

Recall that $\alpha$ is the weight which controls the related magnitude of external and internal interference and $\gamma$ is the weight between the bandwidth and switching delay loss functions, while $\beta$ is used to update the mixed probabilities of the game. Fig. 15- 17 show the delivery ratio obtained with SICA varying $\alpha, \gamma$ and $\beta$, respectively. The figures show the values obtained for a grid network with different number of nodes (depicted with different line types), and using other parameters the same as the parameters considered for the grid scenario.

Fig. 15 shows that SICA performs better for the high values of $\alpha$. This comes from the fact that in our simulation scenario, nodes are prevented from communicating during the time that a channel is occupied by external interference, which has a higher impact than the internal interference on the system performance.

Fig. 16 shows that the performance of SICA is not sensitive to $\gamma$ but values of $\gamma$ in the range of $[0.4,1]$ give better results, which implies that it is more important to give a higher weight to the bandwidth loss functions rather than switching delay loss function. This comes from the fact that we have chosen a Hello interval $\left(T_{H}=20 \mathrm{~ms}\right)$ significantly larger than the switching delay $\left(D_{s}=300 \mu \mathrm{s}\right)$.

Regarding $\beta$, Fig. 17 shows that the best results are obtained with $\beta<0.4$. Recall that choosing a lower value for $\beta$ makes the algorithm to adapt faster to the changes of external interference. 


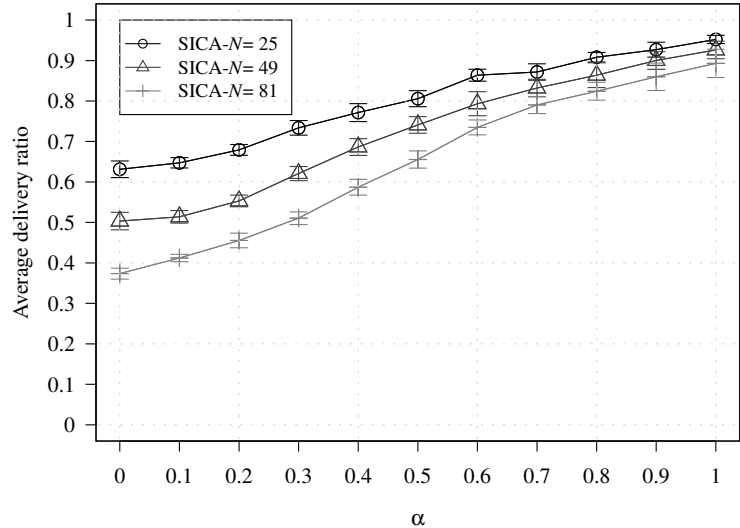

Figure 15: Data delivery ratio vs. $\alpha$

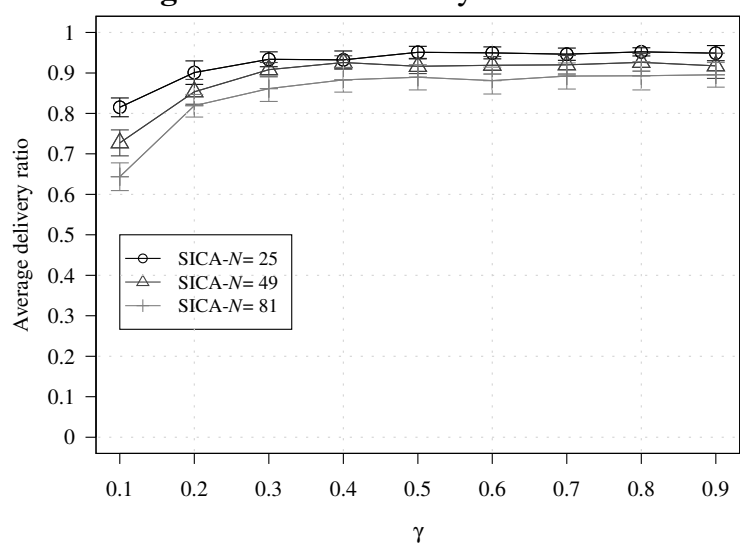

Figure 16: Data delivery ratio vs. $\gamma$

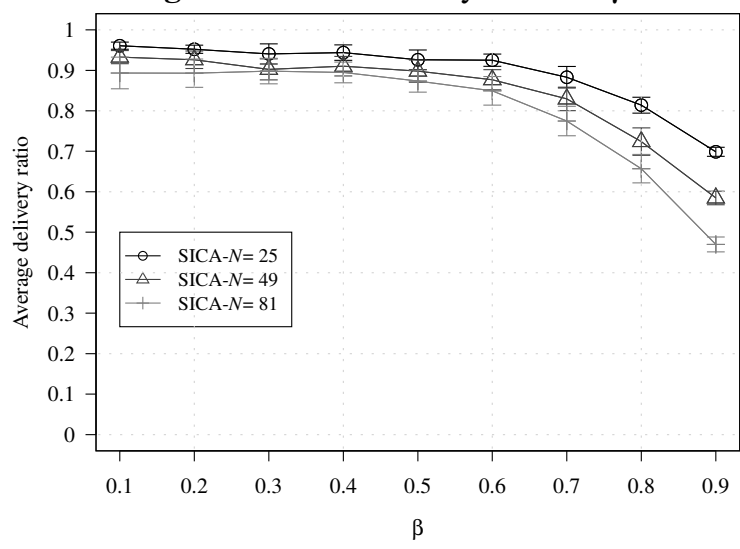

Figure 17: Data delivery ratio vs. $\beta$

\section{Conclusions}

In this paper we have investigated the channel assignment problem in multi-radio wireless mesh networks. We have proposed a new semi-dynamic channel assignment protocol called SICA. We presented a novel formulation for channel assignment problem using game theory and have solved the game using a real time learning mechanism. SICA is a distributed channel assignment and assumes that nodes do not have perfect knowledge about other's nodes strategies. We have done a performance evaluation comparing SICA with other channel assignments mechanisms proposed in the literature. Simulation results show the efficiency of SICA in assigning proper channels to radios by avoiding external interference.

\section{Acknowledgments}

This work was supported by the Spanish government and Generalitat de Catalunya through projects TIN201021378-C02-01 and 2009-SGR-1167, respectively, and by the European Commission through the NoE EuroNF.

\section{References}

Alicherry, M., Bhatia, R. \& Li, L. E. (2006), 'Joint channel assignment and routing for throughput optimization in multiradio wireless mesh networks', IEEE Journal on Selected Areas in Communications 24(11), 1960-1971.

Amiri Nezhad, M. \& Cerdà-Alabern, L. (2010), Utility based channel assignment mechanism for multi radio mesh networks, in 'Proceedings of the 8th ACM international workshop on Mobility management and wireless access', MobiWac '10, ACM, New York, NY, USA, pp. 68-74.

Avallone, S. \& Akyildiz, I. F. (2008), 'A channel assignment algorithm for multi-radio wireless mesh networks', Proceedings of 16th International Conference on Computer Communications and Networks, ICCCN 2007 31(7), 1343-1353.

Bahl, P. \& Chandra, R. (2004), Ssch: Slotted seeded channel hopping for capacity improvement in ieee 802.11 ad-hoc wireless networks, in 'Proceedings of the 10th annual international conference on Mobile computing and networking', pp. 216-230.

Chen, T. \& Zhong, S. (2009), 'Perfectly fair channel assignment in non-cooperative multi-radio multichannel wireless networks', Computer Communications 32(6), $1058-1061$.

Crichigno, J., Wu, M. \& Shu, W. (2008), 'Protocols and architectures for channel assignment in wireless mesh networks', Ad Hoc Networks 6(7), 1051-1077.

Daskalakis, C., Frongillo, R., Papadimitriou, C. H., Pierrakos, G. \& Valiant, G. (2010), On learning algorithms for nash equilibria, in 'Proceedings of the Third international conference on Algorithmic game theory. SAGT'10', pp. 114-125.

Draves, R., Padhye, J. \& Zill, B. (2004), Routing in multiradio, multi-hop wireless mesh networks, in 'Proceedings of the 10th annual international conference on Mobile computing and networking', ACM New York, NY, USA, pp. 114-128.

Felegyhazi, M., Cagalj, M., Bidokhti, S. \& Hubaux, J.-P. (2007), Non-cooperative multi-radio channel allocation in wireless networks, in '26th IEEE International Conference on Computer Communications. IEEE INFOCOM 2007.', pp. $1442-1450$. 
Freund, Y. \& Schapire, R. E. (1996), Game theory, online prediction and boosting, in 'Proceedings of the ninth annual conference on Computational learning theory. COLT '96', ACM, New York, NY, USA, pp. 325-332.

Freund, Y. \& Schapire, R. E. (1999), 'Adaptive game playing using multiplicative weights', Games and Economic Behavior 29(1-2), 79-103.

Fu, W. \& Agrawal, D. (2008), Effective radio partitioning and efficient queue management schemes in a wireless mesh network, in 'Global Telecommunications Conference, 2008. IEEE GLOBECOM 2008. IEEE’, pp. 1 -5.

Gao, L. \& Wang, X. (2008), A game approach for multichannel allocation in multi-hop wireless networks, in 'Proceedings of the 9th ACM international symposium on Mobile ad hoc networking and computing', MobiHoc '08, ACM, New York, NY, USA, pp. 303-312.

Gao, L., Wang, X., Xu, Y. \& Chen, W. (2008), Distributed multi-radio channel allocation in multi-hop ad hoc networks., in 'IEEE International Conference on Communications, 2008. ICC '08', pp. 3156-3160.

Gong, M., Midkiff, S. \& Mao, S. (2009), 'On-demand routing and channel assignment in multi-channel mobile ad hoc networks', Ad Hoc Networks 7(1), 63-78.

Gupta, P. \& Kumar, P. (2000), 'The capacity of wireless networks', IEEE Transactions on Information Theory 46(2), $388-404$.

IEEE-SA (2007), 'Ieee standard for information technologytelecommunications and information exchange between systems-local and metropolitan area networks-specific requirements - part 11: Wireless lan medium access control (mac) and physical layer (phy) specifications'. IEEE Std 802.11-2007 (Revision of IEEE Std 802.11-1999).

IEEE-SA (2008), 'Ieee standard for information technology telecommunications and information exchange between systems local and metropolitan area networks specific requirements part 11: Wireless lan medium access control (mac) and physical layer (phy) specifications amendment 1: Radio resource measurement of wireless lans'. IEEE Std 802.11k-2008 (Amendment to IEEE Std 802.11-2007).

Kim, H.-K., Oh, C.-Y. \& Lee, T.-J. (2009), 'Fair and Collision-Aware Multi-Channel Assignment Based on Game Theory for Wireless Multi-Hop Networks', IEICE Transactions on Communications 92, 1282-1290.

Kim, S.-H. \& Suh, Y.-J. (2008), Rate-based channel assignment algorithm for multi-channel multi-rate wireless mesh networks, in 'Proceedings of the IEEE Global Telecommunications Conf. IEEE GLOBECOM 2008', pp. 1-5.

Kleinrock, L. \& Tobagi, F. (1975), 'Packet switching in radio channels: Part i-carrier sense multiple-access modes and their throughput-delay characteristics', IEEE Transactions on Communications 23(12), 1400-1416.
Kyasanur, P. \& Vaidya, N. H. (2006), 'Routing and linklayer protocols for multi-channel multi-interface ad hoc wireless networks', ACM SIGMOBILE Mobile Computing and Communications Review 10(1), 31-43.

Marina, M. K., Das, S. R. \& Subramanian, A. P. (2010), 'A topology control approach for utilizing multiple channels in multi-radio wireless mesh networks', Computer Networks 54(2), 241 - 256.

Mohsenian-Rad, A. H. \& Wong, V. W. S. (2007), 'Joint logical topology design, interface assignment, channel allocation, and routing for multi-channel wireless mesh networks', IEEE Transactions on Wireless Communications 6(12), 4432-4440.

Murray, D., Dixon, M. \& Koziniec, T. (2007), Scanning delays in 802.11 networks, in 'The 2007 International Conference on Next Generation Mobile Applications, Services and Technologies, 2007. NGMAST '07.', pp. 255 -260 .

Nezhad, M. A. \& Cerdà-Alabern, L. (2011), Adaptive channel assignment for wireless mesh networks using game theory, in 'Proceeding of the IEEE 8th Int Mobile Adhoc and Sensor Systems (MASS) Conf', pp. 746-751.

Nguyen, L. T., Beuran, R. \& Shinoda, Y. (2011), 'An interference and load aware routing metric for wireless mesh networks', International Journal of Ad Hoc and Ubiquitous Computing 7, 25-37.

ns-3 development team (2011), 'Manual: an in-depth coverage of the architecture and core of ns-3 (no. 3.9)'.

$\mathrm{r}$ development core team (2008), $R:$ A Language and Environment for Statistical Computing, R Foundation for Statistical Computing, Vienna, Austria.

URL: $h t t p: / / w w w . R$-project.org

Ramachandran, K. N., Belding, E. M., Almeroth, K. C. \& Buddhikot, M. M. (2006), Interference-aware channel assignment in multi-radio wireless mesh networks, in 'Proceeding of 25th IEEE Int. Conf. Computer Communications INFOCOM 2006', pp. 1-12.

Raniwala, A. \& Chiueh, T.-c. (2005), 'Architecture and algorithms for an ieee 802.11-based multi-channel wireless mesh network', Proceedings of 24th Annual Joint Conference of the IEEE Computer and Communications Societies. INFOCOM 2005. 3, 2223-2234.

Raniwala, A., Gopalan, K. \& Chiueh, T.-c. (2004), 'Centralized channel assignment and routing algorithms for multi-channel wireless mesh networks', SIGMOBILE Mob. Comput. Commun. Rev. 8(2), 50-65.

Shah, I., Jan, S. \& Loo, K.-K. (2010), Selfish flow games in non-cooperative multi-radio multi-channel wireless mesh networks with imperfect information, in "6th International Conference on Wireless and Mobile Communications (ICWMC), 2010', pp. 219-225. 
Si, W., Selvakennedy, S. \& Zomaya, A. Y. (2010), 'An overview of channel assignment methods for multi-radio multi-channel wireless mesh networks', Parallel and Distributed Computing 70, 505-524.

Wooseong Kim, Andreas J. Kassler, M. D. F. \& Gerla, M. (2010), Urban-x: Towards distributed channel assignment in cognitive multi-radio mesh networks, in 'IFIP Wireless Days', Venice, Italy.

Wu, D. \& Mohapatra, P. (2010), From theory to practice: Evaluating static channel assignments on a wireless mesh network, in 'Proceedings of the IEEE INFOCOM, 2010', pp. $1-5$.

Wu, X., Liu, J. \& Chen, G. (2006), Analysis of bottleneck delay and throughput in wireless mesh networks, in 'Proceedings of the IEEE Int Mobile Adhoc and Sensor Systems (MASS) Conf', pp. 765-770.

Yang, P., Qin, G., Yan, Y., Wang, H., Zhang, L., Chen, G. \& Guo, D. (2011), 'On the channel usability of wireless mesh networks: when stability plays with you', International Journal of Ad Hoc and Ubiquitous Computing 8, 64-77. 